\title{
Sex differences in rats' stationary exploration as a function of stimulus and environmental novelty
}

\author{
P. A. RUSSELL \\ University of Aberdeen, Old Aberdeen, AB9 2UB, Scotland
}

\begin{abstract}
Stationary visual scanning and sniffing of novel stimuli were measured using a head-poke response to supplement existing equivocal data on sex differences in exploration. With a short exposure to a novel apparatus, males and females showed a similar frequency of exploratory bouts, but the bouts were longer in females, showing that the sex difference is independent of a simple activity difference. With longer exposure, the difference was reversed, with males "compensating" for their initially lower level. Following familiarization to the apparatus, the sexes did not differ in exploration of an introduced novel stimulus. These results are consistent with males being more susceptible to inhibitory influences, such as disturbance and extreme novelty. Differences in within-trial changes in the level of exploration of a novel stimulus in novel and familiar environments were also observed.
\end{abstract}

Sex differences in behavior in novel situations such as the open field have been reported for rats of various strains (Archer, 1975). In general, females show more exploratory responses, including ambulation, rearing, and sniffing. There appears to be agreement that the difference is linked, at least partly, with circulating estrogen, but the precise behavioral mechanisms involved are unclear. One possibility is that estrogen decreases "emotionality" or "fearfulness" and so produces less inhibition of exploration in the female (Gray, 1971). Alternatively, estrogen may have specific direct effects on exploration (Archer, 1975). A third possibility, also noted by Archer, is that females have a generally higher level of motor activity and that therefore the sex difference is not a reflection of responsiveness to novelty per se. The last hypothesis implies that sex differences will be evident even in response to familiar stimuli and so is consistent with the fact that females are more active in running wheels (Hitchcock, 1925; Martin, 1967) and show more "exploratory" activity in the home cage (Russell, 1973). The issue is further clouded by the possibility that a high level of activity in a novel situation may reflect flight responses and attempts to escape (Welker, 1959; Whimbey \& Denenberg, 1967) rather than, or as well as, exploration. Archer (1975) has suggested that the sexes may differ in the form of their fear-responding, with males tending to immobility and freezing and females to active escape and avoidance.

The present experiments were aimed at clarifying the behavioral nature of sex differences. One reason for the ambiguity of available data is that they come from studies employing measures of activity within a confined

I am indebted to Lorna Rae and Lorraine Craig for testing the subjects and collating the data. area of relatively homogeneous novelty, such as an open field or maze. It is therefore difficult to separate the contributions of exploratory tendencies, flight, and basic activity differences to the activity score. A potentially less ambiguous approach involves the use of clearly localized stimuli differing in novelty, so that approach and avoidance effects can be clearly distinguished and differential preferences for novelty established independent of general activity differences. This approach has clarified the effects of pretest variables such as stress on response to novelty (e.g., Aitken, 1972), but relevant data on sex differences are sparse. Hughes (1968) found that, given a choice of novel and familiar areas, females showed more exploratory responses in the novel area than males, but there was no sex difference in novelty preference as indicated by the time spent in this area. With a similar test, however, Russell (1975) found a stronger preference for novelty in females, suggesting a sex difference in response to novelty independent of activity.

The apparatus used in the present studies requires the rat to make a head-poke response in order to inspect a novel stimulus, so that exploration takes the form of sniffing and visual scanning while the rat is stationary. These behaviors have rarely been examined independently of activity, and where they have the results have been inconsistent. Archer (1974) reported more stationary sniffing in males and Wachs (1973) more visual exploration, which suggests that the conclusion that females are more exploratory may be an oversimplification, since the direction of the sex difference appears to depend on the nature of the response involved. However, in two studies, Russell $(1973,1975)$ found a nonsignificant difference in the direction of greater stationary sniffing in females. 
One aspect of exploration which has been largely ignored in studies of sex differences is habituation. Reports of sex differences sometimes include data on repeated testing in the same situation; for example, Williams and Russell (1972) found similar habituation patterns in males and females for ambulation in an open field over 10 consecutive daily tests. More often, they are based on total scores from a single test (e.g., Archer, 1974; Russell, 1975) and within-test effects are not examined. There is thus no indication of whether intratest habituation is similar in males and females. In addition, as the test is typically short (usually $3.10 \mathrm{~min}$ ), it is unclear how the sex difference is influenced by longer exposure to novelty and whether, for example, it is attenuated or even reversed with continued exposure.

\section{EXPERIMENT 1}

A preliminary experiment checked the existence of sex differences in a situation in which the rat is first permitted to explore, by head poking, a novel stimulus for a short familiarization period, following which this then relatively familiar stimulus is present along with a second, novel one in a test period which permits a comparison of the animal's response to novel and familiar stimuli. In a previous experiment measuring the time spent in a novel area, females had a stronger preference for novelty than did males (Russell, 1975).

\section{Method}

Subjects. Twelve male and 12 female hooded Lister rats, approximately 100 days old, were used. They were selected randomly from a larger group obtained from a supplier at approximately 50 days of age. The rats were caged in like-sex groups of three and maintained on ad-lib pelleted food and water. From supply until testing, the rats were handled only incidentally during the twice weekly cage cleaning. The colony was illuminated $0800-2000 \mathrm{~h}$, and testing was approximately in the middle of the light phase. The female group contained females tested at various stages of the estrus cycle since individual cycles were not monitored and were not synchronized.

Apparatus. The apparatus consisted of the threecompartment box shown in Figure 1. It was $14 \mathrm{~cm}$ deep, painted flat gray, and the top was entirely covered by a removable translucent plastic top which diffused the light from a fluorescent tube positioned centrally $1.5 \mathrm{~m}$ above. The subject compartment (labeled $\mathrm{A}$ in the figure) was just large enough to contain a rat, and the animal could explore the stimulus compartments (B) by inserting its head through the holes $(\mathrm{F})$, which interrupted an infrared beam, permitting the frequency and duration of head poking to be recorded in a separate room. Exploration thus consisted of visual scanning and sniffing of the stimulus compartments. Each hole was $2.5 \mathrm{~cm}$ square, so that only the rat's head could be poked in. The setting of the photobeam $1 \mathrm{~cm}$ into the hole ensured that it could not be broken by the rat pressing its body against the hole. The base of the hole was $5 \mathrm{~mm}$ above the floor of the subject and stimulus compartments, and the photobeam was $2.75 \mathrm{~cm}$ above the floor. Each hole could be blocked by a guillotine door sliding vertically in a slot on the subject-compartment side of the hole so that the door was flush with the wall of this compartment. The doors could be inserted and removed at will, without touching the subject and with minimum disturbance, through slits in the

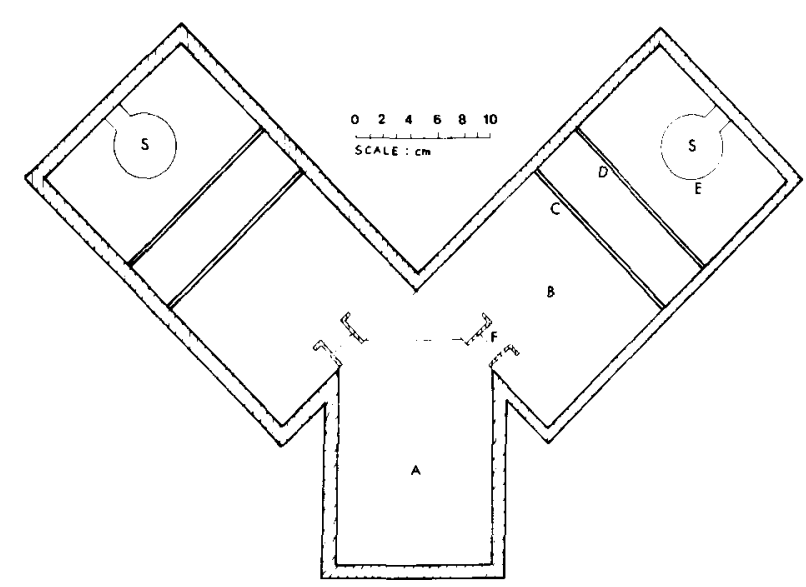

Figure 1. Sectional, schematic plan of the exploration apparatus.

plastic top. The end wall of each compartment (C) was patterned with either horizontal or vertical 5 -mm-wide black and white stripes.

Procedure. Each rat was removed from its cage, carried in the hand to the test room, and placed in the apparatus for $20 \mathrm{~min}$. During the first $10 \mathrm{~min}$ (referred to as familiarization), the hole to one stimulus compartment was blocked. The block was removed for the second $10 \mathrm{~min}$, so permitting access to a novel compartment as well as the now relatively familiar one (test). Feces were removed and the rat compartment wiped with a clean damp tissue between subjects. Within each sex group, half the rats were familiarized to a compartment having a vertically striped end wall and half to a horizontal: within these subgroups, half had the compartment on the left and half on the right. To increase the compartments' visual distinctiveness, the novel compartment end wall had the alternative striped pattern to the familiar one. Test order was random and several rats were tested in succession on any one day.

\section{Results}

Table 1 shows the mean number and duration of head pokes during familiarization. There was no sex difference in the number of head pokes, but the pokes were of longer duration in females $[\mathrm{t}(22)=2.83, \mathrm{p}<.01]$. Corresponding data from the test phase, for the novel and familiar compartments separately, are shown in Table 2. Analysis of variance with sex and compartment as factors revealed that again females had a longer duration $[F(1,22)=4.46, p<.05]$ but not a higher frequency of head poking. The novel compartment elicited both more and longer head pokes than the familiar one $[F(1,22)=22.52$ and $12.37, \mathrm{p}<.001$ and $<.01$, respectively]. There were no Sex by Compartment interactions.

\section{Discussion}

The results confirm the existence of sex differences in stationary visual and olfactory exploration of novel stimuli, with females showing longer, rather than more frequent, bouts of exploration. There is no evidence of a stronger preference for novelty in females, since the absence of Sex by Compartment interactions in the test phase showed that they explored more irrespective of 
Table 1

Experiment 1: Mean Number and Mean Total Duration of Head Pokes During Familiarization

\begin{tabular}{lcc} 
& Males & Females \\
\hline Number of head pokes & 34.1 & 37.9 \\
Head-poke duration $(\mathrm{sec})$ & 35.9 & 57.7 \\
\hline
\end{tabular}

Table 2

Experiment 1: Mean Number and Mean Total Duration of Head Pokes to the Novel and Familiar Compartments During Test

\begin{tabular}{lccccc} 
& \multicolumn{2}{c}{ Novel } & & \multicolumn{2}{c}{ Familiar } \\
\cline { 2 - 3 } \cline { 5 - 6 } & \multicolumn{2}{c}{ Males } & Females & & Males Females \\
\hline Number of head pokes & 24.3 & 27.5 & & 13.6 & 15.0 \\
Head-poke duration $(\mathrm{sec})$ & 30.6 & 55.8 & & 21.6 & 28.8 \\
\hline
\end{tabular}

compartment novelty. It may be argued, however, that $10 \mathrm{~min}$ is a relatively short familiarization period and that, as even the females spent only a mean $10 \%$ of their time exploring the compartment during this period, the "familiar" compartment may well be still sufficiently novel to generate the observed sex difference. In order to establish whether the sex difference persists even to a thoroughly familiar compartment, it is necessary to extend the familiarization period, which was done in Experiment 3.

A further complication with the interpretation of novelty preference effects stems from the fact that since females explore more during familiarization they are presumably more familiar than males with the familiar compartment at the beginning of the test phase, so the choice offered males and females in test may not be equivalent. A similar problem is encountered with locomotor exploration assessed in terms of ambulating, rearing, and sniffing (Russell, 1975). In the present case, however, as there is a single, readily measurable response, the sexes can be matched for the amount of exploration of, and by implication familiarity with, the familiar alternative. This was done in Experiment 2, where matching involved a longer familiarization period for males. This experiment was also designed to provide information on within-trial effects.

\section{EXPERIMENT 2}

\section{Method}

Subjects. Forty male and 20 female Wistar albino rats, aged about 100 days, were used. All other subject details are as in Experiment 1.
Procedure. For 20 males and the 20 females, the procedure was as in Experiment 1. For the second group of 20 males, each rat was matched with one from the female group in a matchedpairs design (= matched males). Matching was achieved by leaving the male in the apparatus during the familiarization phase until he had reached the "target" head-poke time achieved by the corresponding female. Matching was on the basis of duration rather than frequency, in view of the results of Experiment 1. The mean duration of the familiarization phase for matched males was $13 \mathrm{~min} 46 \mathrm{sec}$ with a SD of $6 \mathrm{~min} 58 \mathrm{sec}$. Two matched males which failed to meet their targets within 30 min were replaced. Test order was random, with the stricture that each matched male had to be tested later than his female counterpart (this was not necessarily immediately after). The recording apparatus was arranged to provide a measure of the total headpoke time during successive 2 -min periods.

\section{Results}

The mean head-poke durations during each 2-min period of the familiarization and test phases are shown in Table 3. The familiarization data were analyzed using a repeated-measures-design analysis of variance with Sex and Periods as factors and omitting the matched male data, since the total scores of these rats were identical with those of the females. Females head poked longer than males $[F(1,38)=7.28, p<.02]$, confirming the sex effect obtained with hooded rats in Experiment 1. Head-poking time decreased over periods $[F(4,152)=6.88, p<.001]$, and there was no Sex by Periods interaction. For the test phase, a Group by Compartment by Periods analysis including the matched males revealed significant effects of group $[F(2,57)=$ $4.88, \mathrm{p}<.02]$ and compartment, with the novel comppartment eliciting longer head poking $[F(1,57)=17.17$, $\mathrm{p}<.001]$ and a decline in head-poking time over periods $[F(4,288)=12.92, p<.001]$. There were no interactions. Post hoc mean comparisons revealed that the females head poked longer than both the males $[t(38)=$ $3.22, \mathrm{p}<.01]$ and matched males $(\mathrm{t}=1.98, \mathrm{p}=.05)$, while these last two were not significantly different.

\section{Discussion}

Experiment 2 showed that albino females, like hooded females, explored a novel compartment more than males during both familiarization and test. There was again no evidence of a stronger novelty preference in females, even when the familiar alternative was apparently equally familiar to the two sexes. The within-trial data indicate that exploration of a novel stimulus is highest at the start of an exposure, with a subsequent decline, and this is true for both sexes. There is some indication that the minimum level of exploration occurs after

Table 3

Experiment 2: Mean Total Duration of Head Poking (sec) Over Successive 2-min Periods During Familiarization and to the Novel and Familiar Compartments During Test

\begin{tabular}{|c|c|c|c|c|c|c|c|c|c|c|c|c|c|c|c|}
\hline \multirow[b]{3}{*}{$\begin{array}{l}\text { Males } \\
\text { Matched Males }\end{array}$} & & & & & & \multicolumn{10}{|c|}{ Test } \\
\hline & \multicolumn{5}{|c|}{ Familiarization } & \multicolumn{5}{|c|}{ Novel } & \multicolumn{5}{|c|}{ Familiar } \\
\hline & 12.7 & 8.7 & 11.8 & 7.0 & 8.6 & $\begin{array}{l}12.4 \\
14.8 \\
17 .\end{array}$ & $\begin{array}{r}5.9 \\
9.8 \\
12.6\end{array}$ & $\begin{array}{l}5.4 \\
4.8 \\
7.8\end{array}$ & $\begin{array}{l}8.7 \\
4.9 \\
9.9\end{array}$ & $\begin{array}{r}6.7 \\
6.6\end{array}$ & $\begin{array}{r}8.3 \\
10.8\end{array}$ & $\begin{array}{l}4.5 \\
6.3\end{array}$ & $\begin{array}{l}1.7 \\
4.4\end{array}$ & $\begin{array}{l}5.4 \\
3.4\end{array}$ & $\begin{array}{l}1.8 \\
8.0\end{array}$ \\
\hline
\end{tabular}


about $6-8 \mathrm{~min}$ of a 10 -min exposure, and all groups showed an increase over this level in the last 2-4 min.

\section{EXPERIMENT 3}

The third experiment examined sex differences under extended familiarization and test phases. The necessity of ensuring relatively complete familiarization for the preference test has been noted under Experiment 1. It would also be interesting to know whether, given that with a 10-min exposure to a novel stimulus females explore more but show a similar habituation effect to males, exploration would stabilize with a longer exposure and, if so, whether this stable level would be higher in females or similar in the two sexes. The withintrial data from Experiment 2 also raise the possibility that following an initial decline, exploration may subsequently increase.

\section{Method}

Subjects. Thirty male and 30 female black hooded rats aged about 120 days were used. Other subject details were as in Experiment 1.

Procedure. The procedure was the same as that in Experiment 1 except that familiarization lasted $1 \mathrm{~h}$ and the test phase $30 \mathrm{~min}$. Total head-poke durations were recorded for successive 10 -min periods during familiarization and 2 -min periods during test.

\section{Results}

A Sex by Periods analysis of the familiarization data showed no significant main effect of sex but a significant periods effect $[F(5,290)=5.64, p<.001]$ and a Sex by Periods interaction $[F(5,290)=3.96, p<.01]$, which are illustrated in Figure 2. Overall, exploration time increased across the 1-h exposure, though this is mainly due to the males: females maintained a relatively stable level throughout. As expected from Experiments 1 and
2, females explored more during the first $10 \mathrm{~min}$, but there was then a crossover so that males subsequently explored more. The lack of a significant main sex effect indicates that, overall, males and females explored to about the same extent.

A Sex by Compartment by Periods analysis for the test phase revealed no main sex effect, but did reveal significant effects of compartment $[\mathrm{F}(1,58)=10.69$, $\mathrm{p}<.01]$ and periods $[\mathrm{F}(14,812)=5.64, \mathrm{p}<.001]$. In addition, there was a Compartment by Periods interaction $[F(14,812)=5.74, p<.001]$. These effects are illustrated in Figure 2. Overall, exploration increased across the test phase, though this primarily reflects an increase in head poking to the novel compartment since the times for the familiar compartment remain fairly stable. There is little difference in head-poke time to the two compartments until after $10 \mathrm{~min}$ of exposure, when a marked increase in responding to the novel one occurs. This elevated level persists for the remainder of the session, with a suggestion of a decrease towards the end. The absence of any interactions involving sex shows that these effects are closely similar in males and females.

\section{GENERAL DISCUSSION}

These data on stationary visual and olfactory exploration via head poking supplement existing studies of locomotor exploration in confirming the existence of sex differences. If a short exposure to novelty is considered, females spend more time exploring than males. Here the effect is not simply due to a higher level of motor activity causing females to incidentally head poke more often, since they show the same frequency of exploratory bouts as males, but the bouts are longer. This finding does not preclude the possibility of a more general activity difference between the sexes, which is

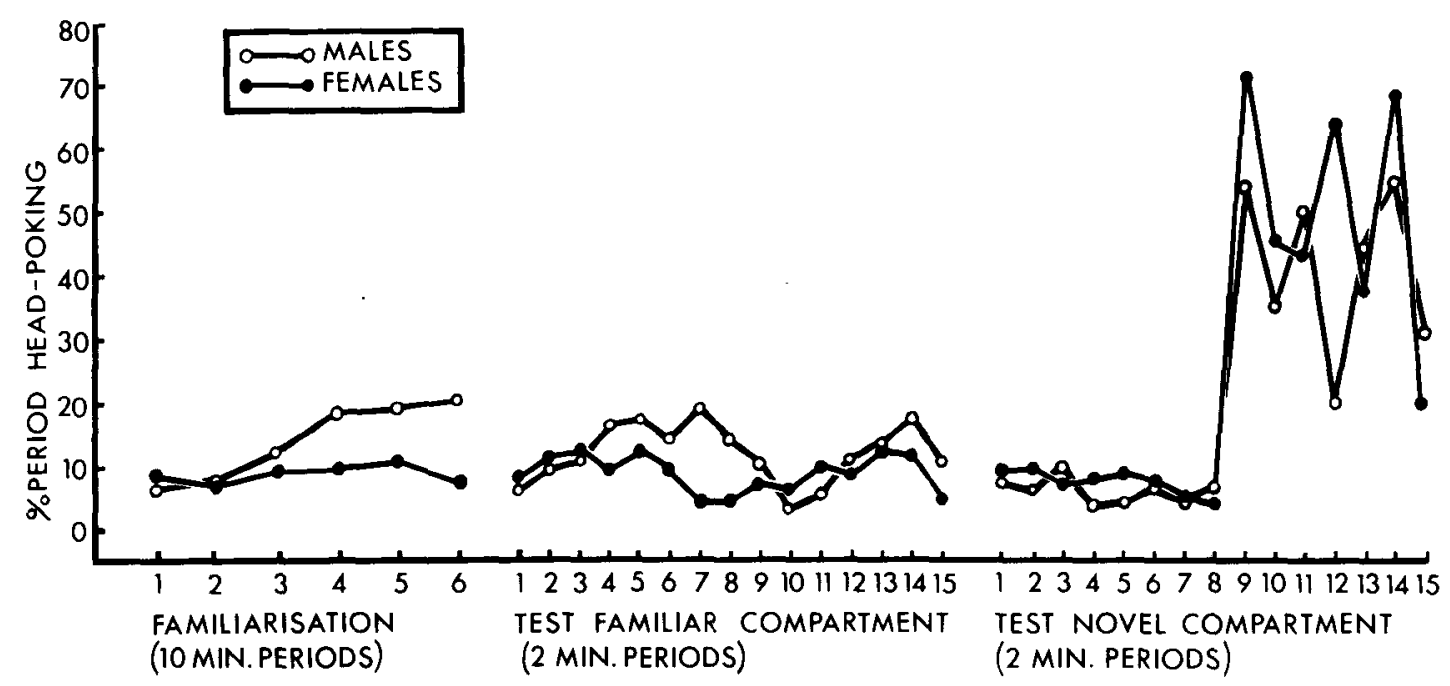

Figure 2. Experiment 3: Percentage of each period spent head poking by males and females during (a) successive 10-min periods of the familiarization phase and (b) successive 2-min periods of the test phase for the familiar and novel compartments separately. 
implied by other findings (see Introduction), but it does suggest that there are sex differences in exploration independent of any such difference.

Extension of the period of exposure to novelty, however, reveals that males subsequently increase their level of exploration with respect to females so that there is a crossover effect, with males and females exploring ultimately for roughly the same length of time. In utilizing relatively short exposures, existing studies appear to have missed this effect. It is clearly necessary to check for the existence of similar within-trial effects with locomotor measures. The finding that females explore a novel stimulus more than males on initial exposure is consistent with the fact that they also spend more time in the novel part of an environment (Russell, 1975). If this is seen as evidence of a stronger preference for novelty in females, then it is clear that with continued exposure this preference is reversed.

The data also suggest that these sex differences are not simply due to differential rates of habituation, since, following familiarization to one stimulus, the introduction of a second stimulus results in females initially exploring the second stimulus more than males if the familiarization period has been short (Experiments 1 and 2) but produces no sex difference if the familiarization period has been long (Experiment 3). In the latter case, within-trial changes in exploration follow an identical pattern in both sexes. This discrepancy is resolved if it is assumed that sex differences appear only following a marked environmental change such as their being placed in a novel test apparatus or at the onset of some other highly novel stimulus. Short-term effects of this kind could arise if males' behavior is more inhibited by disturbance and novelty, as implied by the conventional theory that males are more emotional, and/or if these factors produce different behavior patterns in the two sexes, for example, freezing in males vs. exploration in females. The results of Experiment 3 suggest that once these temporary "inhibitory" effects have subsided, males compensate by exploring more than females. Also, where disturbance effects have dissipated and where the environment is relatively familiar, the introduction of a novel stimulus (Experiment 3, test phase) may not produce any marked sex differences.

The changes in the level of exploratory head poking over the exposure periods are also noteworthy. When the test apparatus is novel, exposure to a novel compartment produces initially a relatively high level of exploration with a subsequent intratrial decline (Experiment 2). This effect is frequently reported for other measures of exploration (e.g., Fowler, 1965, p. 54). With continued exposure (Experiment 3, familiarization phase), exploration may stabilize (females) or increase (males), with the latter effect presumably being related to the initial inhibitory influences operating in males. Experiment 3 (test phase) also shows, however, that when a novel stimulus is introduced into an otherwise relatively familiar environment, there is at first relatively little exploration of that stimulus (no more, in fact, than of a familiar alternative), but the level of exploration subsequently increases. This effect may be similar to that observed in studies of "neophobia," which report initial avoidance of a novel stimulus in a familiar environment (e.g., Mitchell, 1976). These differences may be explainable on the assumption that it is adaptive to explore a totally novel area immediately but to approach a novel stimulus in a familiar environment only with caution.

The main aim of this paper has been to clarify the behavioral nature of the sex difference in response to novelty, but the question of its functional significance may also be considered. Reduced susceptibility of exploration to inhibitory effects in the female compared with the male must be linked ultimately with one or more differential aspects of the male/female roles. One possibility is that it is a reflection of greater general fearfulness in males. Gray (1971) has maintained that a sex difference of this kind stems from the males' participation in dominance interactions (which necessitates fearful behavior patterns), a role not shared by females. A second possibility is that the requirement that the female seek/attract a mate at estrus is favored by a relatively strong, rapid positive response to novelty (and possibly also by higher activity in general). These possibilities are not necessarily mutually exclusive, as selection pressure on a given sex difference may stem from a variety of role requirements. Some light may be shed on the functional aspects of the sex difference by further investigation of its physiological basis and of its relationship to the estrus cycle. Existing evidence relates primarily to the relatively ambiguous open-field ambulation measure. Ambulation is higher at estrus than at diestrus (Birke \& Archer, 1975), and consequently the sex difference in ambulation is reduced (though not eliminated) at diestrus (Quadagno, Shryne, Anderson, \& Gorski, 1972), representing prima facie evidence for the mate-seeking hypothesis. Further studies are necessary to check whether novelty responsiveness per se varies similarly with estrus.

Finally, it may be noted that the problems of the interpretation of differences in exploratory behavior are not specific to sex differences. For example, activity effects and responsiveness to novelty are frequently difficult to disentangle for a variety of early experience factors such as preweaning handling and other environmental modifications and for drug effects. Consideration of visual and olfactory exploration and the adoption of methods described here may also be useful in clarify. ing these effects.

\section{REFERENCES}

ArtKen, P. P. Aversive stimulation and rats' preference for familiarity. Psychonomic Science, 1972, 28, 281-282.

ARChER, J. Sex differences in the emotional behavior of three strains of laboratory rat. Animal Learning \& Behavior, $1974,2,43-48$. 
Archer. J. Rodent sex differences in emotional and related behavior. Behavioral Biology, 1975, 14, 451-479.

Birke, L. I. A., \& ARChER, J. Open-field behaviour of oestrous and dioestrous rats: Evidence against an 'emotionality' interpretation. Animal Behaviour, 1975, 23, 504-512.

Fowler, H. Curiosity and exploratory behavior. New York: Macmillan, 1965.

GrAY, J. A. Sex differences in emotional behaviour in mammals including man: Endocrine bases. Acta Psychologica, 1971, 35, 29.46 .

Hrснсоск, F. A. Studies in vigor: V. The comparative activity of male and female albino rats. American Journal of Physiology, 1925, 75, 205-210.

Hughes, R. N. Behaviour of male and female rats with choice of two environments differing in novelty. Animal Behaviour, 1968, 16. 92-96.

Martin. J. C. Sex differences in the activity wheel and open field as a function of fetal $\mathbf{x}$-irradiation. Psychonomic Science, 1967, 9. 415.416.

MitChell, D. Experiments on neophobia in wild and laboratory rats: A re-evaluation. Joumal of Comparative and Physiological Psychology, 1976, 90, 190-197.

Quadagno. D. M., Shryne, J., Anderson, C., \& Gorski, R. A. Influence of gonadal hormones on social, sexual, emergence, and open-field behaviour in the rat (Rattus norvegicus). Animal Behaviour, 1972, 20, 732-740.

Russell, P. A. Sex differences in rats' stationary-cage activity measured by observation and automatic recording. Animal Leaming \& Behavior, 1973, 1. 278-282.

Russell, P. A. Sex differences in rats' response to novelty measured by activity and preference. Quarterly Journal of Experimental Psychology, 1975, 27, 585-589.

WACHS, T. D. Visual exploratory behavior as a function of early handling, sex and level of auditory prestimulation. Developmental Psychobiology, 1973, 7, 385-392.

WELKER, W. I. Escape, exploratory and food-seeking responses of rats in a novel situation. Journal of Comparative and Physiological Psychology, 1959, 52, 106-111.

Whimbey, A. E., \& Denenberg, V. H. Two independent behavioral dimensions in open field performance. Journal of Comparative and Physiological Psychology, 1967, 63, 500-504.

Williams, D. I., \& Russell, P. A. Open field behaviour in rats: Effects of handling, sex and repeated testing. British Journal of Psychology, 1972, 63, 593-596.

(Received for publication January 31, 1977; revision accepted May 5, 1977.) 